\title{
Die griechische Gewerkschaftsbewe- gung: Protest- und Sozialbewegungen im Kontext der Austeritätspolitik
}

\begin{abstract}
Weshalb gelang es den griechischen Gewerkschaften nicht, die dem Land ab 2010 auferlegten Sparmaßnahmen abzublocken? Welche Veränderungen spielen sich vor dem Hintergrund der harten sozialpolitischen Bedingungen derzeit in der griechischen Gewerkschaftsbewegung ab? Wie interagieren soziale Bewegungen und Gewerkschaften miteinander, inwiefern bereichern sie gegenseitig ihre Programme, Praktiken und Organisationsformen? Diesen und anderen Fragen geht der Beitrag durch eine Bestandsaufnahme und Analyse vieler in Deutschland wenig bekannter Aspekte der gegenwärtigen Auseinandersetzungen in Griechenland nach. ${ }^{1}$
\end{abstract}

MARKOS VOGIATZOGLOU

\section{Einleitung}

Bereits im Vorfeld der Finanzkrise 2008 war das griechische Gewerkschaftssystem von geringer Mitgliederdichte und spärlichen Verbindungen zu anderen gesellschaftlichen Akteuren gekennzeichnet. Die Erfolglosigkeit der Reaktion der Gewerkschaften auf die Griechenland aufgezwungene Austeritätspolitik in den Jahren 2010 bis 2013 macht die mangelnde Schlagkraft der institutionellen Arbeitnehmerorganisationen deutlich. Der vorliegende Beitrag vermittelt einen Einblick in die Veränderungsprozesse, die sich in dieser äußerst schwierigen Situation innerhalb der Arbeiterbewegung abspielen. Dabei liegt der Fokus auf drei Analyseebenen: erstens, auf der mangelnden Fähigkeit (oder Bereitschaft) der etablierten Gewerkschaftseliten, Reformen der Gewerkschaftsstrukturen auf die Tagesordnung zu setzen, die die Verhandlungsmacht der Gewerkschaften im postsozialen Diskussionskontext stärken würden. Zweitens richtet sich die Aufmerksamkeit auf Anstrengungen von Aktivisten, vor allem in Form von Basisgewerkschaften (grassroot unions) mit überwiegend prekär beschäftigten Mitgliedern, an die allgemeine Protestbewegung gegen die Austeritätspolitik anzuknüpfen und deren Agenda um arbeitsbezogene Forderungen zu ergänzen. Drittens geht es um Mischexperimente, die Beschäftigte, Arbeitslose und andere Akteure auf lokaler Ebene zusammenführen, um Solidaritätsnetze für die notleidende Bevölkerung aufzu- bauen. Einige der im Folgenden erwähnten Projekte bewegen sich außerhalb des Blickwinkels traditioneller Gewerkschafts- und Bewegungstheorien. Ihre Aktivitäten stellen de facto eine Neuverhandlung dessen dar, was gewerkschaftliche Organisation bedeutet.

Der Beitrag beruht auf sechsjährigen Forschungsarbeiten zu den griechischen Gewerkschaften. Der Fokus liegt dabei auf den Krisenjahren von 2008 bis 2014, jedoch wird teilweise auch Bezug auf frühere Ereignisse genommen. Die Empirie wurde durch teilnehmende Beobachtung bei verschiedenen gewerkschaftlichen Aktivitäten, 35 Interviews mit Mitgliedern, Führungspersonen und nicht-organisierten Beschäftigten sowie die Auswertung schriftlicher Quellen wie Gewerkschaftspublikationen, Presseveröffentlichungen und Interneteinträgen gewonnen.

Übersetzung aus dem Englischen: Lisa Eskuche. 
TABELLE

Politische Orientierung im Gewerkschaftsbund des Privatsektors (GSEE)

Angaben in absoluten Zahlen

\begin{tabular}{llcc}
\hline Gewerkschafts-Fraktionen & Politische Zugehörigkeit & Stimmen & Sitze \\
\hline PASKE & PASOK (Sozialdemokraten) & 146 & 19 \\
\hline DAKE & ND (Konservative/Rechte) & 103 & 11 \\
\hline DAS-PAME & KKE (Kommunisten) & 94 & 10 \\
\hline AP & SYRIZA (radikale Linke) & 44 & 5 \\
\hline EMEIS & Keine (gemäßigte Linke) & 32 & 3 \\
\hline Sonstige & Außerparlamentarische radikale Linke & 4 & 0 \\
\hline Gesamt & & $\mathbf{4 2 3}$ & $\mathbf{4 8}$ \\
\hline
\end{tabular}

Stamati (2013) errechnet auf Basis von Daten des Jahres 2007 für die Privatwirtschaft, den Organisationsbereich des GSEE, bei einer Zahl von 472.304 Gewerkschaftsmitgliedern einen Organisationsgrad von $18 \%$. Im staatlichen Sektor, wo ADEDY zuständig ist, entsprachen die 311.000 Mitglieder einem Organisationsgrad von rund $60 \%$. Als Gesamtorganisationsgrad gibt Stamati für 2007 rund $28 \%$ an. Die OECD errechnet für 2011 einen Gesamtorganisationsgrad von 25,4\% (OECD 2013).

Der GSEE umfasst drei Organisationsebenen. Die unterste Ebene wird von den (nach Betrieben oder Produktionsbranchen bzw. Berufen organisierten) Einzelgewerkschaften gebildet. Die zweite Organisationsebene besteht aus regionalen Arbeitnehmerzentren und Gewerkschaftsverbänden. Auf der dritten Ebene befinden sich die GSEEFührungsstrukturen (GSEE 2013).

\subsection{Politischer Pluralismus}

\section{Eigenheiten des griechischen Gewerkschaftssystems}

\subsection{Der Einheitsgewerkschaftsverband}

In Griechenland bestehen zwei sich ergänzende gewerkschaftliche Dachverbände: Der Gewerkschaftsbund GSEE vertritt die Arbeitnehmer des Privatsektors, der ADEDY die Staatsbediensteten. Zwar ist Letzterer der zahlenmäßig stärkere Dachverband (Seferiades 1999), doch spielt er bei der Mobilisierung der Arbeitnehmer eine vergleichsweise untergeordnete Rolle. Dies hat damit zu tun, dass entsprechend der griechischen Gesetzgebung Tarifverhandlungen für den staatlichen Sektor unbekannt sind. Der Staat legt Löhne und Gehälter einseitig fest und regelt auch alle übrigen Aspekte der Arbeitsbeziehungen (Ioannou 2000). Die im ADEDY organisierten Gewerkschaften des genau abgegrenzten staatlichen Sektors ${ }^{2}$ sehen normalerweise weder Anreiz noch Gelegenheit, in breiteren gewerkschaftlichen Mobilisierungen eine Führungsrolle zu übernehmen. Traditionell folgt der ADEDY den Mobilisierungsentscheidungen des GSEE und beschränkt sich in eigener Regie auf Kampagnen in Einzelsektoren. Diese Praxis wurde trotz mehrfacher Kürzungen der Beamtengehälter und Entlassung mehrerer Tausend Staatsangestellter auch in den Krisenjahren beibehalten.

Der Dachverband GSEE vertritt etwa 2 Mio. Arbeitnehmer (GSEE 2013). Dies sind nach Angaben der OECD für 2011 44,7 \% der aktiven Bevölkerung (OECD 2012). Für die Beurteilung des Organisationsgrades im Privatsektor stellen diese Zahlen jedoch keine verlässliche Basis dar. Der GSEE vertritt all diese Beschäftigten nur in dem Sinne, dass die vom Dachverband unterzeichneten landesweiten Tarifverträge auch auf alle Arbeitsverträge Anwendung finden, die keiner sonstigen Tarifvereinbarung unterliegen. Der gewerkschaftliche Organisationsgrad ist erheblich niedriger.
Als einzige für die Privatwirtschaft zuständige Dachorganisation des griechischen Gewerkschaftssystems ist der GSEE von seiner politischen Zusammensetzung her zwangsweise pluralistisch angelegt, das heißt, seine Strukturen sind mit Vertretern besetzt, deren politische Überzeugungen das gesamte Spektrum von der außerparlamentarischen Linken bis zur extremen Rechten umspannen. Das zeigt auch die derzeitige Zusammensetzung des Vorstands ${ }^{3}$ (Tabelle 1).

Die den Regierungsparteien PASOK und ND nahestehenden Gewerkschaftsfraktionen halten mit 30 von insgesamt 48 Sitzen eine große Mehrheit. Die Entscheidungsverfahren des GSEE sehen zwar bis zu einem gewissen Punkt ein Mitspracherecht der Minderheitsfraktionen vor, doch letzten Endes wird die politische Strategie des Bundes mehrheitlich beschlossen. Wichtig ist in diesem Zusammenhang die Anmerkung, dass die Gewerkschaften der ersten und zweiten Organisationsebene nicht an die Entscheidungen des GSEE gebunden sind; sie können jederzeit auch ohne Zustimmung oder Unterstützung des GSEE Kampfmaßnahmen ausrufen. Dieses relativ hohe Maß an Unabhängigkeit ist fest in der Geschichte der griechischen Gewerkschaftsbewegung verankert.

\subsection{Das Wahlsystem des GSEE}

Den arbeitsrechtlichen Vorschriften entsprechend werden die in den GSEE-Kongress zu entsendenden (und somit an der Wahl des Bundesvorstands beteiligten) Gewerkschaftsvertreter in den Vorstandswahlen der Einzelgewerkschaften

2 Halböffentliche und kürzlich privatisierte Unternehmen sowie in staatlicher Hand befindliche öffentliche Infrastrukturgesellschaften sind im GSEE organisiert.

3 Nach den Ergebnissen des letzten GSEE-Generalkongresses (März 2013). 
bestimmt. Da die Wahlen der primären Gewerkschaften nicht zeitgleich mit dem Bundeskongress stattfinden, kann es vorkommen, dass in den Kongress entsandte Vertreter mehrere Jahre zuvor gewählt wurden. In normalen Zeiten beeinflusst diese Eigenheit des griechischen Gewerkschaftssystems die Wahlergebnisse des Bundeskongresses nur unwesentlich. Da die politische Landschaft Griechenlands aber seit 2010 massive Veränderungen durchgemacht hat, spiegelt sich der allgemeine Meinungswandel nur unzulänglich in der Zusammensetzung des GSEE-Vorstands wider. Bei genauerer Betrachtung fällt auf, dass die der sozialdemokratischen PASOK nahestehende Gewerkschaftsfraktion rund $42 \%$ aller Sitze hält. Nach Meinungsumfragen Anfang 2014 käme die PASOK in den nächsten Wahlen jedoch nur auf 3,5 bis 5,5\%. Auf die der linksradikalen Partei SYRIZA nahestehende AP-Fraktion entfallen dagegen derzeit nur $11 \%$ der Sitze, obwohl die Partei in den landesweiten Wahlen 2012 beachtliche $27 \%$ erzielte. Wie im folgenden $\mathrm{Ab}$ schnitt näher ausgeführt, hat diese Eigenheit des Gewerkschaftssystems zur Vergrößerung der bereits beträchtlichen Kluft zwischen den Gewerkschaftseliten und der Basis beigetragen.

\section{Gewerkschaftsführung: Ein Teufels- kreis aus Organisationsdefizit und gesellschaftlicher Unglaubwürdigkeit}

\subsection{Die Gewerkschaftsreaktion auf die Austeritätspolitik}

Als die sozialdemokratische Regierung Griechenlands im April 2010 das mit der sogenannten Troika aus Europäischer Zentralbank (EZB), Europäischer Union (EU) und Internationalem Währungsfonds (IWF) unterzeichnete Finanzhilfeabkommen bekannt gab, wurde klar, dass eine lange Zeit rigider Sparpolitik bevorstand. Die Arbeitnehmervertreter reagierten mit einem nach griechischen Maßstäben konventionellen Handlungsrepertoire. Als Hauptkampfmaßnahme setzte der GSEE (mit Unterstützung des ADEDY) auf eine Welle vierundzwanzigstündiger, landesweiter Generalstreiks. Der erste dieser Streiks fand am 5. Mai 2010 statt; im selben Jahr folgten elf weitere. In der Zwischenzeit hat die Zahl der Generalstreiks die 50 überschritten. An den Streiktagen füllen sich die Straßen Athens und anderer griechischer Großstädte mit Zigtausenden von Demonstranten. Zusammenstöße mit der Polizei und andere Äußerungen von Gewalt sind keine Seltenheit.

Konkrete Verbesserungen haben die Massenkundgebungen jedoch kaum bewirkt. Ausnahmslos alle Sparmaßnahmen wurden vom Parlament angenommen, trotz des zweimaligen Zusammenbruchs der griechischen Regierung innerhalb von vier Jahren und obwohl die PASOK, eine der beiden Hauptgruppierungen des bis dahin bipolaren Parteiensystems, von der politischen Bühne gefegt wurde.

Damit nicht genug: Die Gewerkschaftsführungen mussten sich dem weitverbreiteten Vorwurf stellen, ihre Reaktion sei unzureichend beziehungsweise dem Problem unangemessen gewesen. Nicht weniger als 95,2\% der Befragten meinten 2013 in einer Umfrage, die Gewerkschaften hätten „sehr wenig oder nichts” unternommen, um die soziale Kahlschlagpolitik abzuwenden (Lykavitos 2013).

\subsection{Die Hintergründe des Teufelskreises}

Die Erklärung für die oben genannte Entwicklung ist sowohl in der sozialen Unglaubwürdigkeit der Gewerkschaftseliten als auch in organisatorischen Mängeln der Gewerkschaften $\mathrm{zu}$ suchen, die schon lange vor der Krise bestanden. Aus Sicht der griechischen Arbeitnehmerschaft haben vierundzwanzigstündige Generalstreiks eher symbolischen als praktischen Wert. Selbst in Zeiten relativen Arbeitsfriedens waren zwei bis drei Generalstreiks pro Jahr nichts Ungewöhnliches. Die rein quantitative Steigerung in den Krisenjahren wurde für unzureichend erachtet und als Beweis für die Unfähigkeit des GSEE gewertet, sein Repertoire zu modernisieren. Andererseits war die von vielen radikalen $\mathrm{Ba}$ sisparteien vorgeschlagene Alternative eines landesweiten, unbefristeten Generalstreiks nicht nur deshalb undurchführbar, weil die Verbandsspitzen nicht bereit waren, sich auf eine solche „Mutter aller Schlachten“ einzulassen, sondern auch, weil der Gewerkschaftsbewegung für eine solch lang anhaltende, ressourcenintensive Maßnahme die nötigen organisatorischen Voraussetzungen fehlten.

Von der bereits erwähnten geringen Gewerkschaftsdichte einmal ganz abgesehen, hatten die griechischen Gewerkschaften mit umfangreichen Arbeitskämpfen schlicht und einfach keine Erfahrung. Die letzten dokumentierten branchenübergreifenden Arbeitskämpfe von längerer Dauer spielten sich in den 1980er und Anfang der 1990er Jahre ab (Ioannou 2000), als die Gewerkschaften deutlich stärker waren als heute. Seitdem war die Gewerkschaftsaktivität auf der Makroebene nicht über die Vertretung der Arbeitnehmer in den Strukturen des "sozialen Dialogs“ hinausgegangen (Daskalakis 1995).

Eine umfassendere gewerkschaftliche Mobilisierung hätte zudem einen entsprechend starken gesellschaftlichen Rückhalt erfordert. In der Gesellschaft dominierte aber eine ziemlich feindliche Haltung gegenüber den Gewerkschaftsspitzen. Diese waren in den Augen der Bevölkerung nahezu genauso diskreditiert wie die Politiker. In einer Meinungsumfrage im Jahr 2011 sagten nur 7\% der Befragten, dass sie Vertrauen in die Gewerkschaftsführer hätten - nur zwei Prozentpunkte mehr als bei der Frage nach dem Vertrauen in Parlamentsabgeordnete (Laoutaris 2011). Diese Ablehnung gegenüber der Führung der griechischen Gewerkschaften ist nicht mit der Wertschätzung von Gewerkschaften als solchen gleichzusetzen. Doch hatten bereits in einer im Herbst 2010 in 27 EU-Ländern durchgeführ- 
ten Umfrage zum Vertrauen in Institutionen auf die Frage, ob sie Gewerkschaften „eher vertrauen“ oder „eher nicht vertrauen“ würden, nur $29 \%$ der griechischen Befragten „eher vertrauen“ geantwortet (Europäische Kommission 2011). Ein Wert, der deutlich unter dem EU-Durchschnitt von $38 \%$ lag und nur in Bulgarien, Slowenien und Rumänien unterschritten wurde.

Um es abzukürzen: Der Führung des GSEE war entgangen, dass im radikal veränderten gesellschaftspolitischen Umfeld der Krisenjahre qualitative Antworten wichtiger gewesen wären als rein quantitative. Hingegen erkannten in gewissem Umfang die Aktivisten und Gruppierungen aus den sozialen Bewegungen ${ }^{4}$ diese Notwendigkeit. Nach einem ganzen Jahr vergeblicher „traditioneller“ Mobilisierungen (Mai 2010 - Mai 2011) revidierte die griechische Anti-Austeritätsbewegung ihre Strategien und Taktiken von Grund auf: Erstens wurde das „Platzbesetzungs-Modell“ des Arabischen Frühlings und der spanischen 15M-Bewegung in angepasster Form übernommen (Sergi/Vogiatzoglou 2013), zweitens flossen Ressourcen in den Aufbau sogenannter „sozialer Solidaritätsstrukturen” (Mattoni/Vogiatzoglou 2014). Diese Solidaritätsstrukturen bilden die Grundlage der experimentellen Organisationsprojekte der Arbeiterbewegung, die in Abschnitt 5 behandelt werden.

\section{Die Basisgewerkschaften: Prekär Beschäftigte gegen Austeritätspolitik}

\subsection{Gewerkschaften für prekär Beschäftigte}

Vor dem Hintergrund eines traditionell schwach ausgebildeten Sozialschutzes brachte die Einführung flexibler Arbeitsverträge Mitte der 1990er Jahre eine neue Kategorie von Beschäftigten hervor, die sowohl Flexibilität als auch Schutzlosigkeit auszeichnete (Triantafyllou 2008). Diese prekär Beschäftigten waren zudem nicht gewerkschaftlich organisiert, entweder, weil sie in neu entstandenen Betrieben arbeiteten, oder weil die Gewerkschaften in Betrieben mit überwiegend flexibler Belegschaft schwach waren (Vogiatzoglou 2010). Seit Anfang der 2000er Jahre sind jedoch in verschiedenen Wirtschaftssektoren, unter anderem dem Fernmeldewesen, dem Gastgewerbe, der Gebäudereinigung und den Kurierdiensten, gewerkschaftliche Organisierungsbestrebungen zu verzeichnen. Die Initiatoren dieser Organisationsansätze trafen sich teils im Betrieb, teils außerhalb. K. G., ein Mitglied des später als sektorale Basisgewerkschaft konstituierten Kollektivs audiovisueller Techniker Diakoptes, beschreibt den Gründungsvorgang seiner Organisation folgendermaßen:

„Die Gruppe nahm ihre Arbeit Anfang 2009 auf, nachdem wir uns während der Dezember-Revolte ${ }^{5}$ kennenge- lernt hatten [...] Uns verband die Tatsache, dass wir in der gleichen Branche, im Showbusiness, tätig waren. [Auf dieser Basis] haben wir die Initiative Diakoptes gegründet“ [Gespräch mit K. G., 2010].

Diese sowohl auf Betriebsebene als auch branchenweit tätigen Basisorganisationen vertreten in erster Linie prekär Beschäftigte; der Einfachheit halber bezeichne ich sie im Folgenden deshalb als Gewerkschaften prekär Beschäftigter (GPB). Ein Mitglied der Gewerkschaft des Telekom-Unternehmens Wind beschreibt die Mitgliedschaft seiner Organisation folgendermaßen:

„Unsere Firma beschäftigt nicht nur flexible Arbeitnehmer, sondern auch eine relativ hohe Anzahl an fest angestellten Verwaltungsmitarbeitern und Fachkräften. Trotzdem habe ich festgestellt, dass praktisch alle an den Versammlungen teilnehmenden Gewerkschaftsmitglieder prekär beschäftigt sind. [...] Woran das liegt, weiß ich nicht - die Mitarbeiter mit festeren Arbeitsverhältnissen sind wohl nicht so stark daran interessiert, mitzumachen. Sie glauben wohl, dass ihre persönlichen Anstrengungen zur Sicherung ihrer Arbeitsplätze ausreichen. So gut haben wir [anderen] es nicht, wissen Sie ..." [Gespräch mit N.A., 2012].

Im Laufe der 2000er Jahre zogen die Gewerkschaften der prekär Beschäftigten eine Reihe sehr einfallsreicher Aktionen durch. 2009 wurde dann mit der Einrichtung einer horizontalen Koordination der Athener Basisgewerkschaften (Syntonismos Protovathmion Somation) ein neues Kapitel aufgeschlagen (Vogiatzoglou 2011). Andere Städte folgten dem Athener Beispiel. Zum ersten Mal seit Jahrzehnten wurden damit Anstrengungen unternommen, die Arbeitnehmerschaft über die Strukturen des GSEE hinaus zu organisieren. Dabei ist jedoch zu beachten, dass „über den GSEE hinaus“ weder die Absicht meint, sich mit dem Gewerkschaftsbund anzulegen, noch eine Distanzierung vom Gewerkschaftssystem insgesamt bedeutet. Zwar reicht die Position der Führungsspitzen der GPB von schlichter politischer Opposition bis zu völligem Ignorieren der Strategien und Vorgehensweisen des Bundes, doch ist die Mehrheit der genannten Organisationen in den GSEE-Strukturen vertreten. Um es mit den Worten von K. B., Mitglied der Gewerkschaft der lohnabhängigen Techniker mit Sitz in Athen, zu sagen:

„Unser Verhältnis zum GSEE ist schlecht, wir begegnen dem Bund mit einer gewissen Distanz. Dennoch beteiligen

4 Gemeint sind Organisationen wie z. B. die Athener Aftonomo Steki (Autonome), SKYA (Versammlung zur Verbreitung der Kämpfe), Diktyo Koinonikis Ypostiriksis Metanaston (Netzwerk zur Unterstützung von Migranten) oder Antieksousiastiki Kinisi (Antiautoritäre Bewegung).

5 K. G. spielt hier auf die gewalttätigen Unruhen im Dezember 2008 an, die durch die Tötung eines 15-jährigen Schülers durch einen Angehörigen der Polizeikräfte ausgelöst wurden. 
wir uns an seinen Strukturen. Wenn Sie nämlich jemanden im Vorstand eines lokalen Arbeitnehmerzentrums oder Verbandes haben, ist dieser Vertreter befugt, bei Arbeitsstreitigkeiten, beispielsweise wenn jemandem gekündigt wird, zu intervenieren" [Gespräch mit K. B., 2010].

Nach Meinung unseres Gesprächspartners lässt sich diese scheinbar widersprüchliche Haltung also auf die Notwendigkeit zurückführen, für die eigenen Mitglieder adäquate Leistungen zu erbringen - ohne Institutionalisierung keine Beteiligung an den Tarifverhandlungsverfahren des jeweiligen Unternehmens oder Wirtschaftszweigs.

\subsection{Die Beteiligung der GPB an der Anti- Austeritätsbewegung}

Wichtig ist in diesem Zusammenhang, dass die Gewerkschaften der prekär Beschäftigten sofort eine intensive Vernetzung mit anderen Bewegungsorganisationen begannen. Ausschlaggebend war hierbei einerseits die relativ schwache Verhandlungsposition der Gewerkschaften prekär Beschäftigter, andererseits der Umstand, dass die Gründer und Führungsspitzen dieser Gewerkschaften bereits an Bewegungen beteiligt gewesen waren und folglich in diesem Bereich über beträchtliche Erfahrungen und Kontakte verfügten. Wie ich an anderer Stelle dokumentiert habe, zeigten die GPB ein deutlich größeres Interesse daran, mit gleichartigen Gewerkschaften und nicht arbeitsbezogenen Bewegungsgruppen zusammenzuarbeiten als mit den etablierten Gewerkschaftsorganisationen (Vogiatzoglou 2011).

Diese engen Verbindungen stellten sich als äußerst nützlich heraus, als sich die Widerstandsbewegung gegen die Austeritätspolitik formierte. Die Athener Koordination organisierte eine umfassende Gewerkschaftsversammlung und rief eigenständig (d.h. unabhängig vom GSEE und mit separatem Treffpunkt) zu Generalstreikdemonstrationen auf (aformi 2010). Dabei lautete die Botschaft an potenzielle Teilnehmer: Man muss sich nicht mit dem (als regierungsfreundlich und zahnlos geltenden) Gewerkschaftsbund identifizieren, um sich an Protesten gegen die Austeritätspolitik zu beteiligen. K. V., Führungsmitglied einer an den Versammlungen der Koordination beteiligten Basisgewerkschaft, erinnert sich:

„In den Austeritätsjahren war die Koordination unübersehbar und entscheidend an allen größeren Kampfmaßnahmen beteiligt - von der Gesamtheit der Generalstreiks bis zur Besetzung des Athener Syntagma-Platzes. Infolgedessen wird sie in weiten Kreisen der Arbeitnehmerschaft nun als Gegenpol zur arbeitgeberfreundlich gesinnten, bürokratischen Gewerkschaftspolitik des GSEE gesehen" [Gespräch mit K. V., 2013].

Dem Aufruf folgte bald auch eine Vielzahl anderer Organisationen, darunter kleine Linksparteien, Studierendenverbände und sogar Nichtregierungsorganisationen (NGO). Das Ergebnis war erstaunlich: Während die GPB-Aufrufe Zehntausende, gelegentlich sogar Hunderttausende mobilisierten und zur treibenden Kraft aller Anti-Austeritätsde- monstrationen wurden, gelang es dem GSEE nie, mehr als zehntausend Teilnehmer am Ausgangsplatz ihrer Kundgebungen zu versammeln.

Andererseits riefen die GPB ihre Mitglieder aber auch zu Aktionen nicht arbeitsbezogener Bewegungen auf und unterstützten Letztere technisch und mit Fachwissen. Ein Beispiel hierfür ist das bereits erwähnte Kollektiv audiovisueller Techniker Diakoptes, das zur Bereitstellung eines Medienteams am Syntagma-Platz beitrug. Freiberufliche Programmierer und Netzwerktechniker der TelekomGewerkschaften sorgten während der Platzbesetzung für Internet-Verbindungen. Die Gewerkschaft der Kellner und Köche von Athen richtete 2010 die erste Streik-Suppenküche ein, gefolgt von vielen anderen Kollektiven, die heute auch die verarmte Bevölkerung mit kostenlosen Mahlzeiten versorgen. Und schließlich ist es kaum verwunderlich, dass unter den Aktivisten der (im Abschnitt 5 behandelten) Versuchslabors arbeitnehmerischer Organisation zahlreiche Mitglieder, Führungskader und Gründer von GPB anzutreffen sind.

\subsection{Grenzen des Handlungsspielraums der Basisgewerkschaften}

Trotz dieser vielversprechenden Entwicklungen stehen die GPB vor verschiedenen Problemen bei der Ausdehnung ihrer Aktivitäten und Reichweite. Als eine der größten Herausforderungen stellt sich für Basisgewerkschaften die Frage, wie sich eine breite und flexible gewerkschaftsübergreifende Koordinationsstruktur konsolidieren lässt, ohne die lebendige interne Demokratie zu gefährden. Einige Teilnehmer an der Athener Koordination äußern die Sorge, dass diese selbst Gefahr läuft, sich zu bürokratisieren. $\mathrm{Zu}$ dieser Entwicklung trägt die Rivalität unter den vertretenen politischen Parteien bei - wobei zu beachten ist, dass die meisten griechischen Gewerkschaftsorganisationen sehr stark, wenn auch pluralistisch, politisiert sind. K. V. stellt fest:

„Ich fürchte, wir werden auch zunehmend bürokratisch. SYRIZA versucht ständig, die Kontrolle über die Koordination zu gewinnen. Und wir [die außerparlamentarische Linke] operieren als Fraktion. [...] Das Ganze wirkt eher wie eine Top-Down-Initiative. Wir sollten dafür kämpfen, dass die Grundmerkmale, die uns als Basisorganisation auszeichnen, nicht verloren gehen " [Gespräch mit K. V., 2013]

Obwohl die GPB wirksamer als der GSEE in der Lage gewesen sind, ihr eigenes Handlungsrepertoire einzusetzen, ist ihre Kreativität bisher nicht über Aktionen hinausgegangen, die man als „traditionell“ bezeichnen könnte. Da die GPB hauptsächlich innerhalb der Betriebe arbeiten, ist ihr Repertoire auch um arbeitsplatzbezogene Aktivitäten zentriert, wie die Organisation von Streiks, Streikposten sowie die Verbreitung von Informationsmaterial unter den Beschäftigten. Ihre Aktivitäten außerhalb der Betriebe gehen selten über die Teilnahme an Protesten und Streikdemonstrationen hinaus. In dieser Hinsicht wird es für außen- 
stehende Beobachter schwieriger, zwischen GPB und den traditionellen Gewerkschaften Unterschiede auszumachen, abgesehen von der radikaleren Rhetorik der Ersteren. Zudem scheint sich nach einem sprunghaften Anstieg sowohl der Anzahl der Gewerkschaften als auch ihrer Mitgliedschaft im Zeitraum 2008-2009 die zahlenmäßige Entwicklung stabilisiert zu haben (Vogiatzoglou 2011) und in Einzelfällen sogar rückläufig zu sein. Diese Tatsache hängt möglicherweise mit dem negativen Effekt der hohen Arbeitslosigkeit zusammen, der die Mitgliedszahlen drückt (ca. $28 \%$ der gesamten Arbeitnehmerschaft und über $60 \%$ der Jugendlichen sind ohne Beschäftigung). Überdies steht außer Zweifel, dass sich das Format der GPB rein organisatorisch nicht dazu eignet, massenhaft Arbeitslose zu mobilisieren. Im nächsten Abschnitt geht es um die Versuche der griechischen Arbeitnehmerbewegung, diese schwierige Problematik zu lösen.

\section{Die „Versuchslabors“ - Ausblicke auf die Zukunft der griechischen Arbeiter- bewegung}

In den vorstehenden Abschnitten habe ich mehrfach auf eine Reihe von Mischansätzen der griechischen Arbeiterbewegung Bezug genommen, die ich als "Versuchslabors“ bezeichnen möchte. Das Interessante an diesen Experimenten ist, dass sie nicht auf die Diskussion abstellen, welche gewerkschaftlichen Aktivitäten angesichts der Notlage der griechischen Gesellschaft am zweckdienlichsten wären, sondern bestrebt scheinen, die Inhalte der Gewerkschaftsbewegung insgesamt neu zu bestimmen. Zum Beispiel indem sie scheinbar selbstverständliche Dinge wie den Begriff Arbeitsplatz und die Machtverhältnisse der Arbeitswelt hinterfragen und Vorschläge unterbreiten, die auf radikale Veränderungen der Form und der Inhalte gewerkschaftlicher Organisation hinauslaufen.

Ich ordne diese Experimente in zwei große Kategorien: Zunächst betrachte ich Projekte, für die ich eine mittel- und langfristige Überlebenschance sehe, dann solche ohne Aussichten auf Fortbestand.

\subsection{Experimente mit Zukunftsaussichten jenseits der Krise}

An erster und möglicherweise prominentester Stelle unter den „Versuchslabors“ stehen die sogenannten ArbeiterKlubs (Ergatikes Leshes), die in letzter Zeit in verschiedenen Stadtteilen Athens aus dem Boden geschossen sind. Ähnlich wie die worker center, ihre US-amerikanischen Vorläufer (Fine 2006), wollen diese Vereine den Arbeitskampf über die Betriebe hinaustragen. Mit ihrer flexiblen Struktur und ihrem lokalen Fokus schaffen sie es, zwei Bevölkerungs- gruppen, die sich dem Einfluss der etablierten Gewerkschaften entziehen - Mitarbeiter von Kleinstunternehmen und Arbeitslose -, anzusprechen und mit Veranstaltungen und Aktivitäten in ihr Klublokal zu locken. Um es mit den Worten eines Mitglieds des Arbeiterklubs Nea Smyrni (WCNS) auszudrücken:

„Der Arbeiter-Klub hofft, zu einer „Stadtgewerkschaft” zu werden, will aber die gewerkschaftliche Arbeiterbewegung nicht aus dem Arbeitsleben verdrängen, sondern sie ergänzen. Gleichzeitig hat der Klub sich den Schulterschluss zwischen Beschäftigten und Arbeitslosen im städtischen Umfeld auf die Fahnen geschrieben" [Gespräch mit WCNS, 2012].

An zweiter Stelle befinden sich die besetzten und ,zurückgewonnenen“ Betriebe. Hier sind vor allem das Werk VIOME in Thessaloniki und die staatliche Hörfunk- und Fernsehanstalt (ERT) in verschiedenen griechischen Städten zu nennen. In beiden Fällen wurde die Produktion vom jeweiligen Arbeitgeber stillgelegt und von der Belegschaft wieder aufgenommen. VIOME verkündete unlängst die Absicht, den Vertrieb seiner Erzeugnisse durch die Gründung einer Kooperative auf rechtlich sichere Beine zu stellen. Das ERT-Rundfunk- und Fernsehprogramm wird seit der Zwangsräumung der Athener Zentrale von über ganz Griechenland verstreuten Studios ausgesendet. Im internationalen Kontext sind Belegschaftsübernahmen natürlich nichts Neues (man denke nur an Ereignisse der frühen 2000er Jahre in Lateinamerika); dennoch sollte man nicht vergessen, dass neuartige Vorschläge dieser Art in den Diskussionen der griechischen Arbeiterbewegung bisher nie zur Sprache gekommen waren. VIOME-Mitarbeiter Makis Anagnostou beschreibt die Organisation der von der Belegschaft übernommenen Fabrik folgendermaßen:

„Wir haben beschlossen, alle Mitarbeiter absolut gleichzustellen - gleicher Lohn für alle, unabhängig von Tätigkeit, die der Einzelne versieht. Weiterhin lautete der Entschluss: eine Unternehmensaktie und eine Stimme pro Mitarbeiter. [...] Und schließlich wurde entschieden, dass die Betriebsleitung jederzeit abberufen werden kann. Gleiches gilt für den Gewerkschaftsvorstand. So und nicht anders sollte es unserer Meinung nach in einem von der Belegschaft geführten kooperativen Unternehmen zugehen“ (Papadatos-Anagnostopoulos/Vogiatzoglou 2013).

Zu guter Letzt verdienen die in jüngster Zeit zu Dutzenden entstandenen Kooperativen Erwähnung. Sie bieten eine breite Palette von Produkten und Dienstleistungen an - von Landwirtschaftserzeugnissen bis zur Computerreparatur, von Kurierdiensten bis zu Restaurants und Bars. Auch in diesem Zusammenhang ist zu berücksichtigen, dass die Erfahrung genossenschaftlicher Bewegungen in Griechenland fehlt. Entsprechend wenig ist man sich der Dynamik bewusst, die beispielsweise spanische und italienische Kooperativen in Einrichtungen zur Ausbeutung billiger Arbeitskräfte und zur Steuerhinterziehung verwandelt hat. Der Beitrag der Genossenschaften und besetzten Betriebe besteht vor allem darin, dass sie die Diskussion über demo- 
kratische Mitsprache in der Arbeitswelt und über das Eigentum an den Produktionsmitteln aus ihrem Dornröschenschlaf geweckt haben.

\subsection{Experimente ohne Zukunftsaussichten}

Die Krise nicht überleben werden meiner Meinung nach die Strukturen sozialer Solidarität, die in den letzten zwei Jahren als gesellschaftliche Reaktion auf die humanitären Folgen der Sparpolitik entstanden sind. Zu verstehen sind darunter Krankenhäuser, Apotheken, Lebensmittelläden und Suppenküchen sowie Elektrikerteams, die zahlungsunfähige Haushalte, denen der Strom abgeschnitten wurde, schwarz wieder ans Netz anschließen. Alle diese Einrichtungen verlassen sich auf Freiwillige und bieten ihre Dienste und Waren kostenlos an. Ich habe sie aus zwei Gründen in diesen Beitrag aufgenommen: Erstens sind sie dank ihrer flexiblen und direktdemokratischen, auf Plenumsbeschlüssen basierenden Entscheidungsstruktur deutlich effizienter als der bürokratische, korrupte und schwachbrüstige griechische Sozialstaat. Zweitens erinnert ihr mutualistischer Ansatz an die Frühzeiten der Gewerkschaftsbewegung, als die Praxis noch viel stärker im Fluss war und sich noch keine endgültige Vorstellung von den gemeinsamen Pflichten und Zielsetzungen der Arbeiterschaft durchgesetzt hatte.

Diesen Experimenten wird vermutlich deshalb eine kurze Lebensdauer beschieden sein, weil die Freiwilligen (mit Ausnahme einer kleinen radikalen Minderheit) selbst nicht den Drang verspüren, ihre Aktivitäten über das unbedingt Erforderliche hinaus zu verlängern. Sie verstehen ihre Aktionen als Notmaßnahmen angesichts einer außergewöhnlichen, durch Sozialabbau bedingten Situation. Ihr gemeinsames politisches Ziel ist (ungeachtet unvermeidlicher Meinungsverschiedenheiten bezüglich der optimalen Vorgehensweise und des erwarteten Ergebnisses) die Wiederherstellung sozialer Fürsorge für die Schwachen der Gesellschaft in der einen oder anderen Form, jedoch nicht auf der Grundlage wohltätiger, unentgeltlich erbrachter Leistungen.

\section{Probleme, Herausforderungen und Schritte in die Zukunft}

Im vorliegenden Beitrag habe ich die vielfältige Art und Weise dargelegt, in der sich Arbeitnehmerorganisationen und aus sozialen Bewegungen entstandene Einrichtungen vor dem Hintergrund der düsteren gesellschaftspolitischen Lage des heutigen Griechenlands gegenseitig befruchtet haben (oder auch nicht) - in ihren Zielsetzungen, ihrem gemeinsamen Kampfrepertoire und der Wahl ihrer Strategien. Trotz einiger sehr inspirierender praktischer Erfahrungen ist es unwahrscheinlich, dass sich einer der beschriebenen Vorschläge und eines der experimentellen Projekte in seiner heutigen Gestalt als langfristiges Rezept für die
Wiederbelebung der griechischen Arbeiterbewegung durchsetzen werden. Die etablierten Gewerkschaften haben ihr Innovationspotenzial schon lange erschöpft und ihre Unfähigkeit bewiesen, sich an dringende Erfordernisse des rapiden Zeitwandels anzupassen. Zwar sind sich die Spitzenfunktionäre der damit verbundenen Herausforderungen an die Gewerkschaftsorganisation bewusst, doch sträuben sie sich, den Ruf nach drastischen strukturellen Veränderungen zu unterstützen. ${ }^{6}$ Die gewerkschaftlichen Basisorganisationen können die Arbeitslosen nur unzulänglich erreichen und sind aufgrund des gewerkschaftsfeindlichen Umfelds äußerem Druck ausgesetzt. Die Arbeitervereine sind noch selten und dienen dem eigenen Selbstverständnis nach der Ergänzung anderer Gewerkschaftsstrukturen. Erfahrungen in anderen Teilen der Welt bieten Grund zu der Annahme, dass die Kooperativen und besetzten Betriebe allenfalls am Rande des Wirtschaftssystems überleben werden.

Man darf ebenfalls nicht vergessen, dass viele der erwähnten Projekte inoffizieller Natur sind, da das griechische Arbeitsrecht die entsprechenden Unternehmensformen und Aktivitäten nicht vorsieht. Die Reform des griechischen Gewerkschaftssystems kann sich folglich nicht nur auf den Anstoß von „unten“ verlassen: Will man die erstarrten Strukturen der etablierten Gewerkschaften wieder beweglich machen und die interessantesten der laufenden Experimente auf kohärente und sinnvolle Art und Weise in diese Strukturen integrieren, werden Gesetzesinitiativen unumgänglich sein. Ich halte es für höchst unwahrscheinlich, dass die offiziellen Gewerkschaftsorganisationen ihre Funktion völlig einbüßen und von der Bildfläche verschwinden. Keiner der Hauptakteure, und ganz sicher weder die Regierung noch die Arbeitgeber, würden sich für diesen Ausgang entscheiden. Ein kraftloses Gewerkschaftssystem ist allemal der Unruhe vorzuziehen, welche das Fehlen einer offiziellen Arbeitnehmervertretung hervorrufen würde. Dennoch scheinen in Anbetracht des beschriebenen „Teufelskreises” und der im griechischen Arbeitsleben seit mehreren Jahrzehnten fehlenden Strukturen sozialen Dialogs nur zwei Wege nach vorn zu gehen: Entweder begnügen sich die Gewerkschaften mit einer noch marginaleren gesellschaftlichen Rolle als bisher oder sie packen die Ausgestaltung und Umsetzung der unumgänglichen strukturellen Veränderungen an. Dieser Wandel wird sich in einem Umfeld abspielen müssen, in dem die Verflechtungen zwischen Gewerkschaften und sozialen Bewegungen von facettenreichen Prozessen und hoher Komplexität geprägt sind.

Aus dem Gesagten lässt sich die Schlussfolgerung ziehen, dass die wissenschaftliche Untersuchung des Falls Griechenland Phänomene einbeziehen muss, die der Arbeits-

6 Eine eingehendere Analyse findet sich beispielsweise in Lanara 2012. Lanara ist im GSEE für internationale Beziehungen zuständig. 
forschung gelegentlich als irrelevant gelten. Nur dann ergibt sich ein vollständiges Bild. Diese Forderung wirft jedoch weitere Fragen auf: Wie ließe sich ein solches Forschungsfeld abstecken? Welche Vorgänge sollten berücksichtigt, welche können als unerheblich außer Acht gelassen werden? Und schließlich: Auf welcher Grundlage ließen sich Vergleiche zu anderen Ländern ziehen, wenn die zu berücksichtigenden Variablen auch die Eigenheiten der jeweiligen sozialen Bewegungen beinhalten? Diesen und ähnlichen Fragen wird die Sozialforschung künftig nicht aus dem Weg gehen können.

\section{LITERATUR}

aformi (2010): Grassroots' unions coordination: An interview with Panagiotis Sotiris, aformi.wordpress.com, http://aformi.wordpress.com/2010/06/17/ (letzter Zugriff: 16.07.2010)

Daskalakis, D. (1995): Collective autonomy in the contemporary industrial society: The collective bargaining experience in European countries, Thessaloniki Europäische Kommission (2011): Eurobarometer 74, Herbst 2010. Die öffentliche Meinung in der Europäischen Union, TNS opinion \& social, Brüssel

Fine, J. R. (2006): Worker centers: Organizing communities at the edge of the dream, New York

General Confederation of Greek Workers (GSEE) (2013): The structure of GSEE, http://www.gsee.gr/left_menu_files/left_m_p2c.php?p_id=6\&p2_id=1 (letzter Zugriff: 01.04.2013)

Ioannou, C. (2000): Change and continuity in Greek industrial relations: The role of and impact on trade unions, in: Waddington, J./Hoffmann, R. (Hrsg.): Trade unions in Europe: Facing challenges and searching for solutions,

Brüssel, S. 277-304

Koukoules, G./Tzanetakos, V. (1986): Syndicalist movement 1981-1986: The great lost opportunity, Athen

Lanara, Z. (2012): Trade unions in Greece and the crisis: Friedrich-Ebert-Stiftung, http://library.fes.de/pdf-files/id/ipa/09012.pdf (letzter Zugriff: 01.04.2014) Laoutaris, G. (2011): The blurry image of local administration, http://laoutaris.

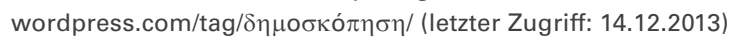

Lykavitos (2013): Opinion poll: What the Greeks are afraid of, http://lykavitos. gr/archives/77016, (letzter Zugriff: 14.12.2013)

Mattoni, A./Vogiatzoglou, M. (2014): “Today, we are precarious. Tomorrow, we will be unbeatable": Early struggles of precarious workers in Italy and Greece, in: Royall, F./Chabanet, D. (Hrsg.): From silence to protest: International perspectives on weakly resourced groups, London/New York (im Erscheinen) Organisation for Economic Co-operation and Development (OECD) (2012): OECD.StatExtracts., 2012, from http://stats.oecd.org (letzter Zugriff: 30.01.2012) Organisation for Economic Co-operation and Development (OECD) (2013): Population, employment (national concept), employment by industry (domestic concept): Greece, Quarterly National Accounts 2013/3, Paris

Papadatos-Anagnostopoulos, D./Vogiatzoglou, M. (2013): \#syndicalism 2013: An interview with the Wage Earners Technicians' Union, Red Notebook, Athen, http://www.rednotebook.gr/details.php?id=9014 (letzter Zugriff: 21.05.2013) Seferiades, S. (1999): Low union density amidst a conflictive contentious repertoire. Flexible labour markets, unemployment, and trade union decline in contemporary Greece: European University Institute, EUI Working Paper SPS 99/6, Florenz

Sergi, V./Vogiatzoglou, M. (2013): Think globally, act locally? Symbolic memory and global repertoires in the Tunisian uprising and the Greek anti-austerity mobilizations, in: Fominaya, C. F./Cox, L. (Hrsg.): Understanding European movements. New social movements, global justice struggles, anti-austerity protest, S. 220-236, London

Stamati, A. (2013): Greece: Industrial relations profile, Brüssel, http://www.eurofound.europa.eu/eiro/country/greece_3.htm

Triantafyllou, C. (2008): Precarious work in Greece, in: Network Of Refugees' And Immigrants' Social Support (Hrsg.): Discussion on precarious forms of employment, Athen, S. 12

Vogiatzoglou, M. (2010): Precarious workers' unions in the Greek syndicalist movement, University of Crete

Vogiatzoglou, M. (2011): Precarious workers' unions in the aftermath of a student rebellion, ECPR General Conference, Reykjavik

\section{AUTOR}

MARKOS VOGIATZOGLOU ist Wissenschaftler am Europäischen Hochschulinstitut, Florenz. Arbeitsschwerpunkte: Soziale Bewegungen, Arbeitsstudien, prekäre Beschäftigung, vergleichende Politik.

markos.vogiatzoglou@eui.eu 